Supporting Information

\title{
Effects of Cholesterol on the Partitioning of a Drug Molecule in Lipid Bilayers
}

Running title: Cholesterol Regulates Amantadine Partition in Membrane

Yuqin Yang ${ }^{1}$, Hao Dong ${ }^{1,3, *}$, and Huan-Xiang Zhou ${ }^{2, *}$

${ }^{1}$ Kuang Yaming Honors School, Nanjing University, 210023 Nanjing, China

${ }^{2}$ Department of Chemistry and Department of Physics, University of Illinois at Chicago, Chicago, IL 60607

${ }^{3}$ Institute for Brain Sciences, Nanjing University, 210023 Nanjing, China

*Correspondence e-mail: donghao@nju.edu.cn, hzhou43@uic.edu 

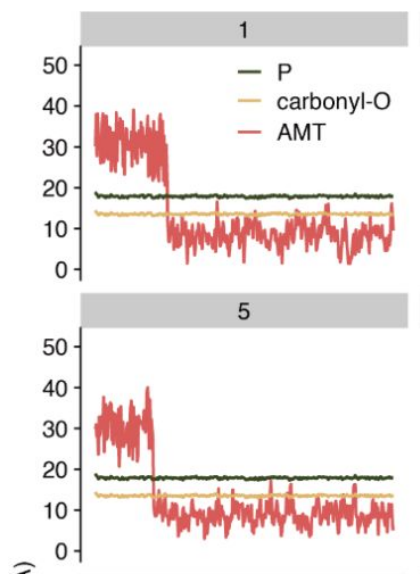

$\underset{\mathrm{N}}{\mathrm{S}}$
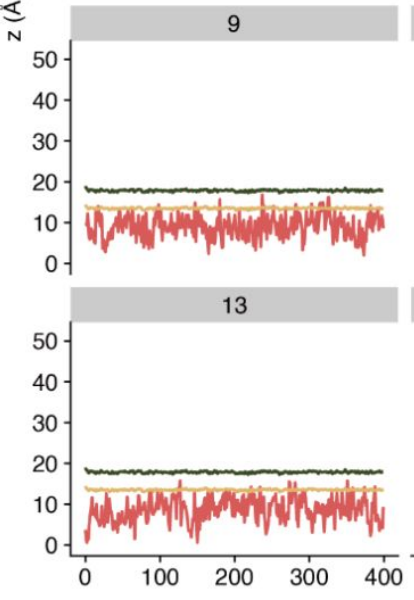
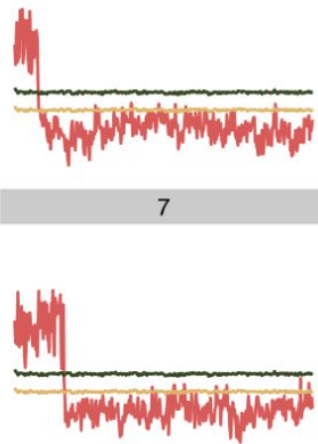

11

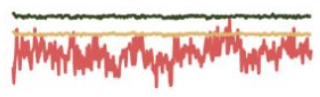

14

10
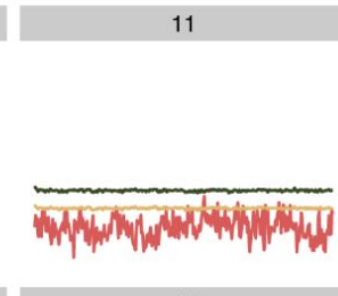

15

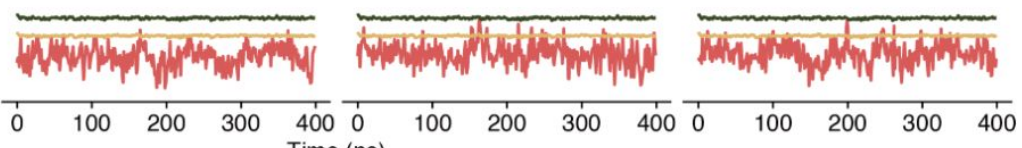

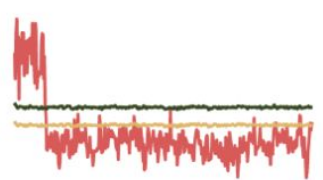

8
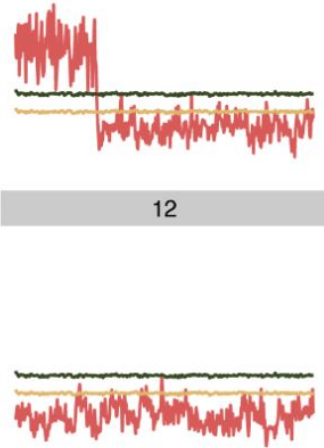

16

Figure S1. The $\boldsymbol{z}$ positions of AMT in the simulations with pure DMPC membrane $\left(x_{\text {chol }}=0 \%\right)$. 


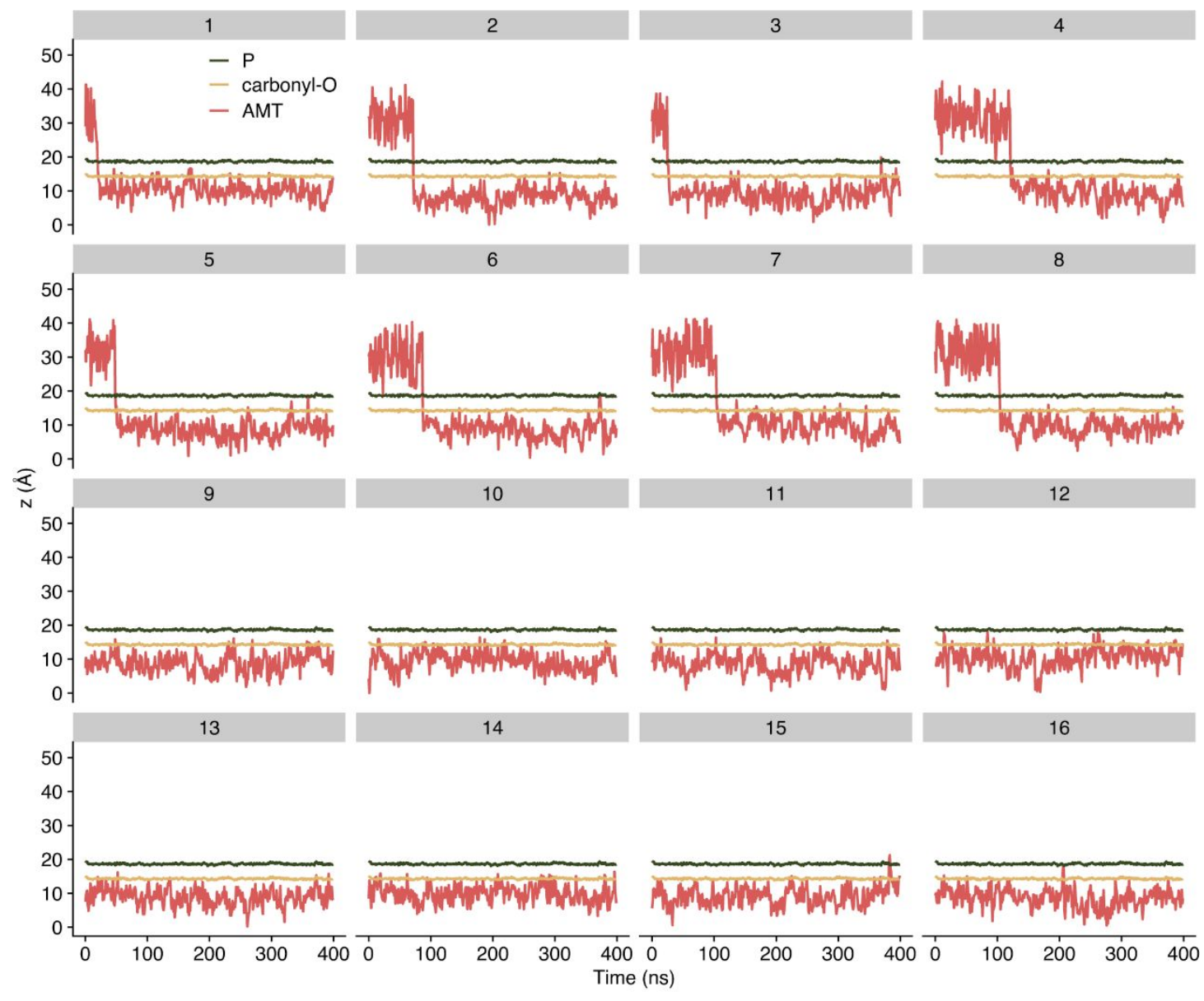

Figure S2. The $z$ positions of AMT in the simulations with DMPC/cholesterol membrane $\left(x_{\text {chol }}=7 \%\right)$. 


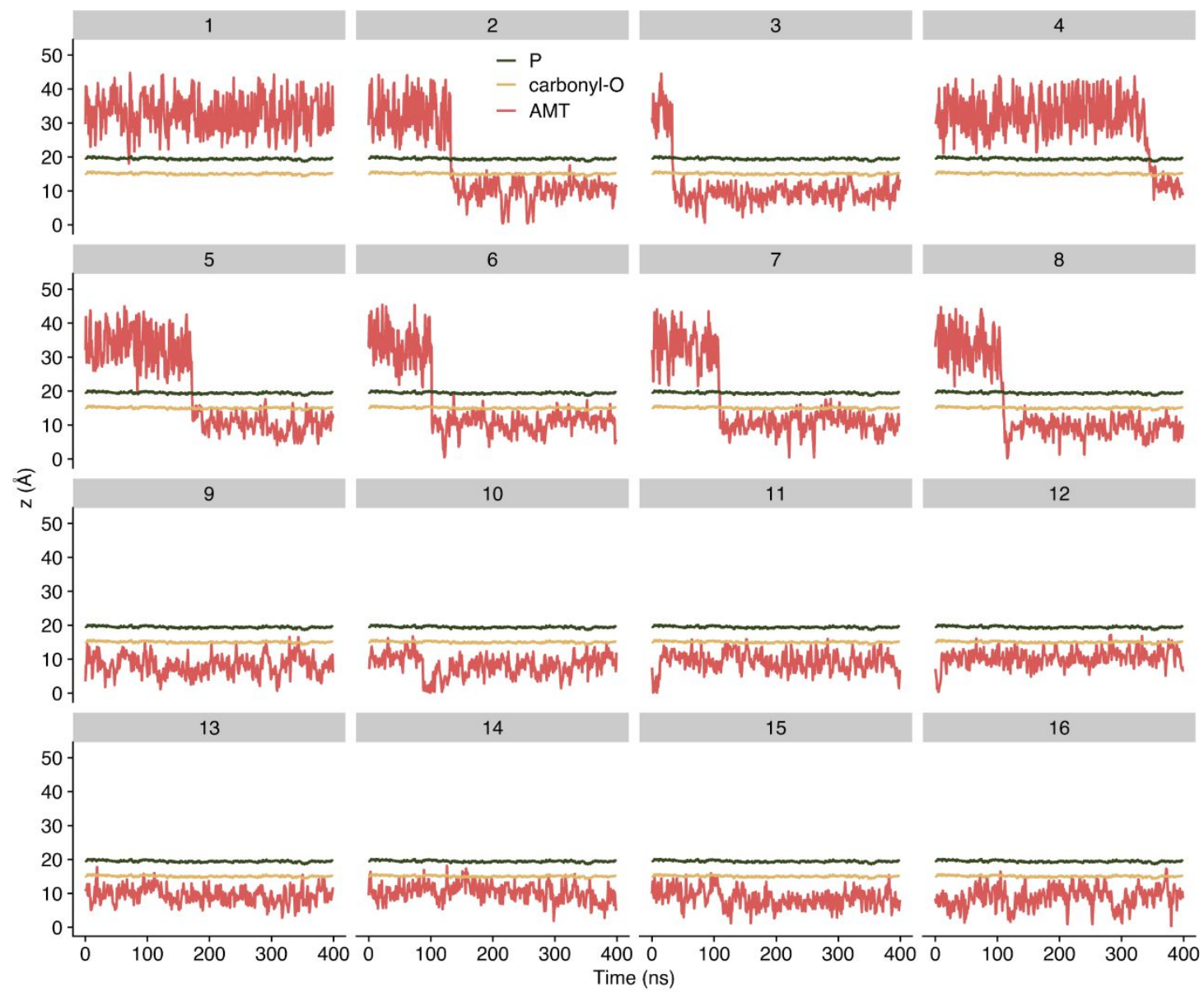

Figure S3. The $z$ positions of AMT in the simulations with DMPC/cholesterol membrane $\left(x_{\text {chol }}=14 \%\right)$. 

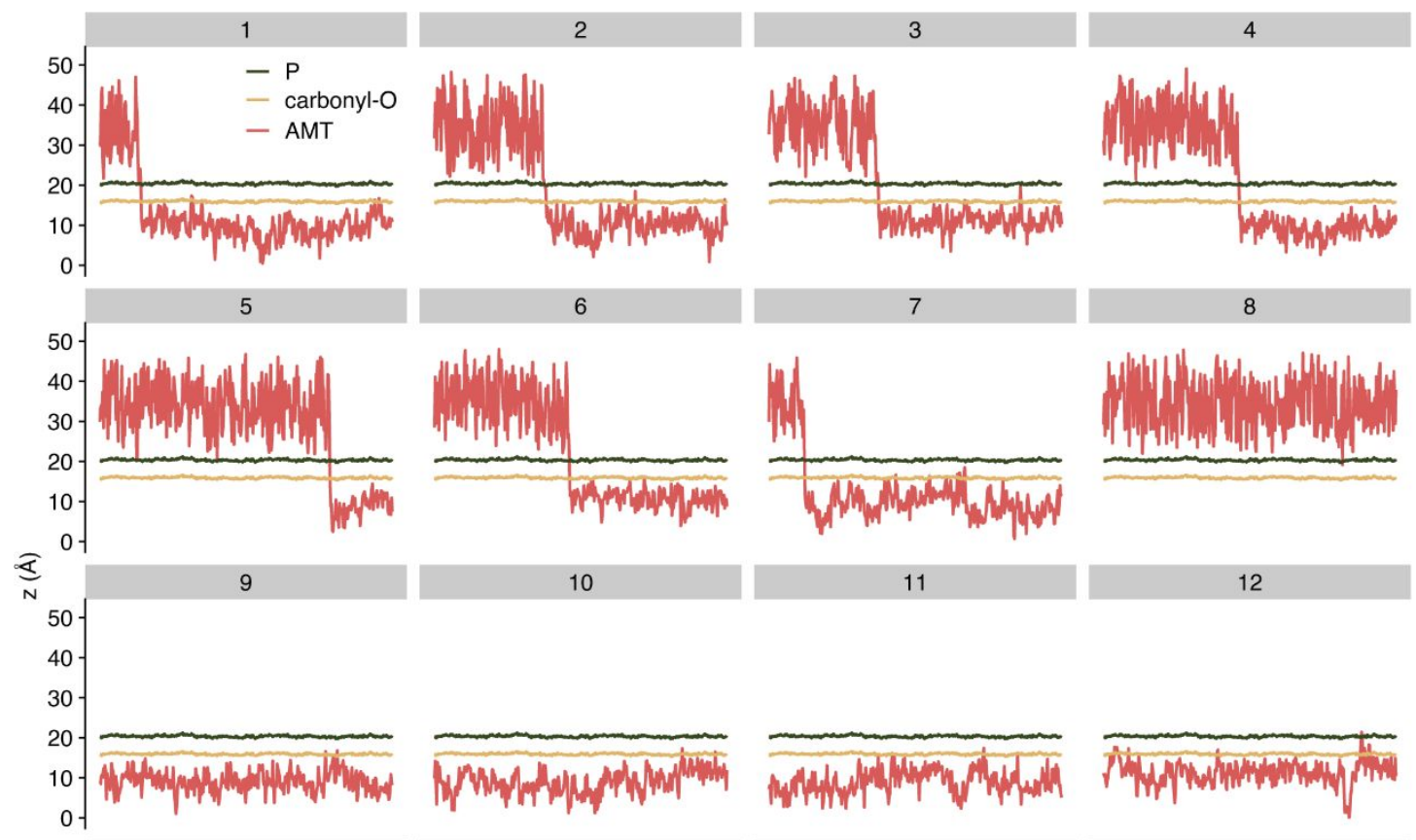

11

12
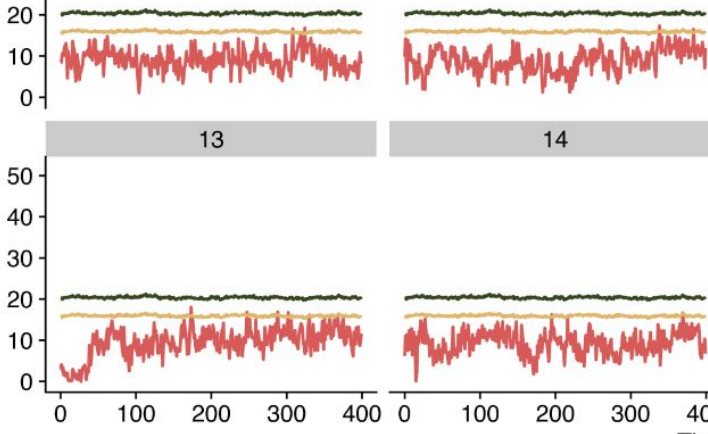

14
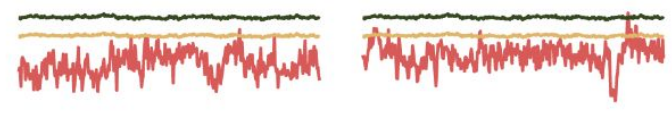

15

16

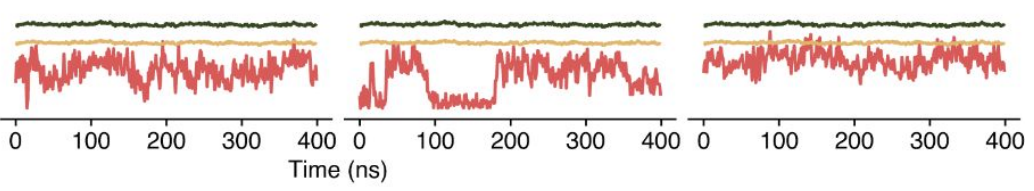

Figure S4. The $z$ positions of AMT in the simulations with DMPC/cholesterol membrane $\left(x_{\text {chol }}=21 \%\right)$. 

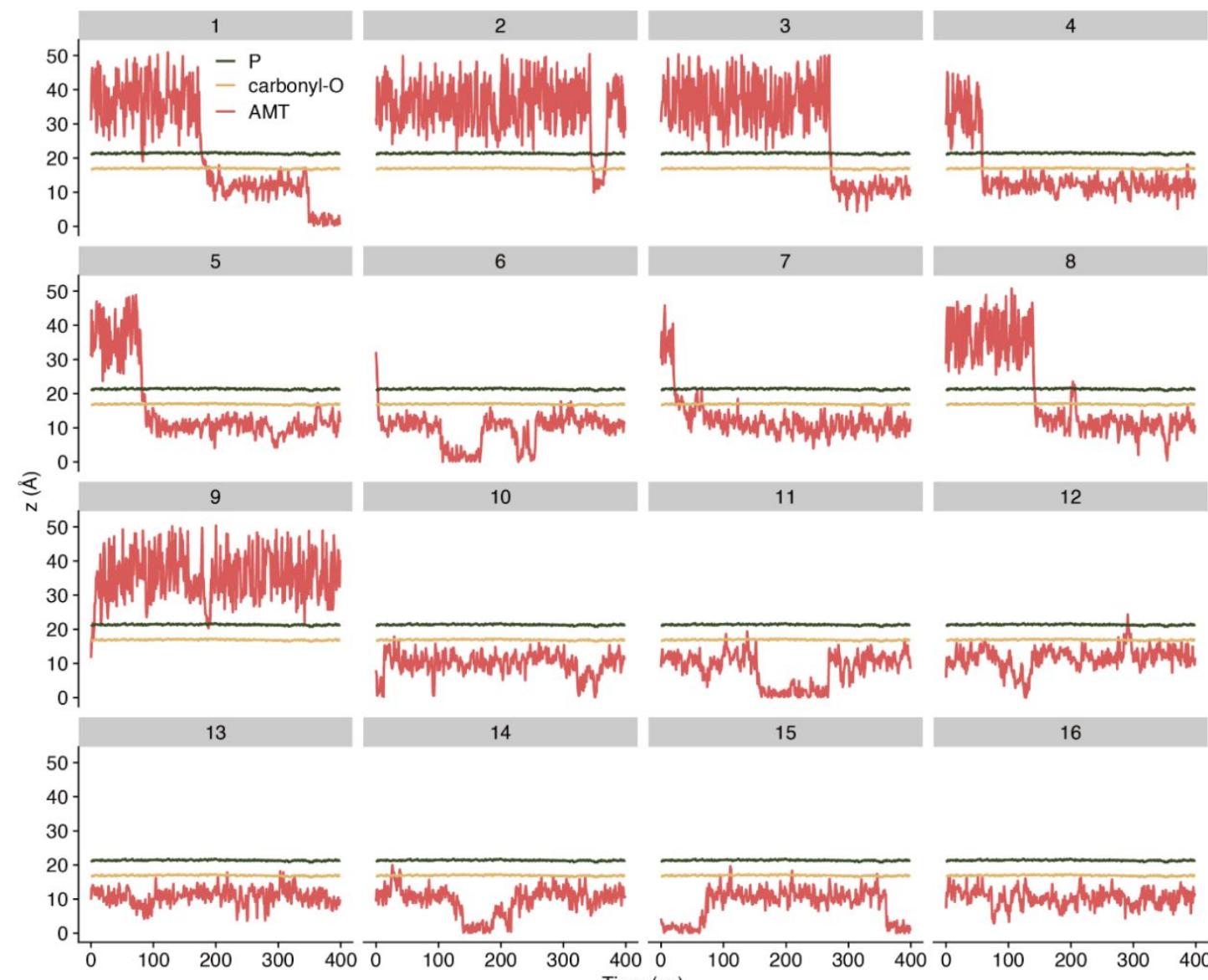

15

16

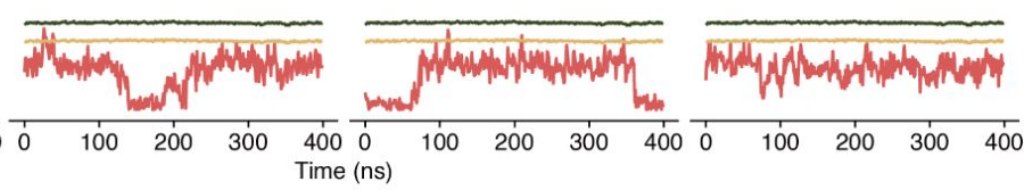

Figure S5. The $z$ positions of AMT in the simulations with DMPC/cholesterol membrane $\left(x_{c h o l}=32 \%\right)$. 
A

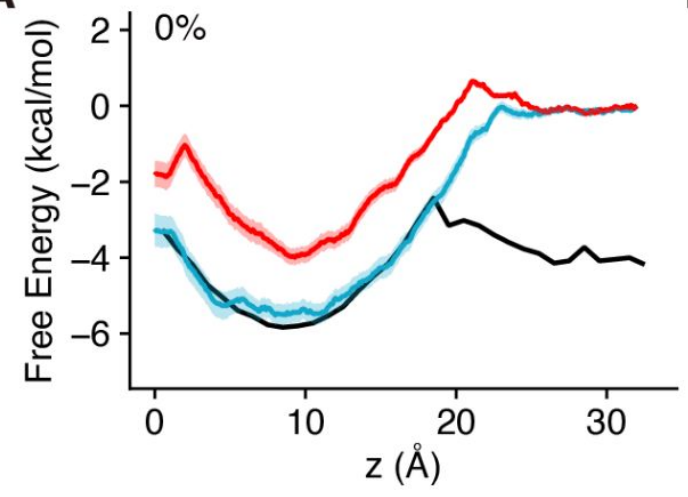

B

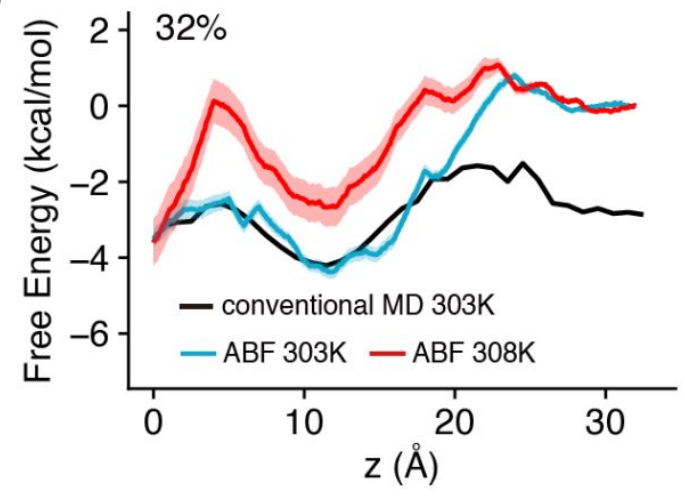

Figure S6. Comparison of PMF profiles obtained from conventional MD simulations and by the ABF method. (A) $x_{\text {chol }}=0 \%$; (B) $x_{\text {chol }}=32 \%$. Density profiles from conventional MD simulations are converted to PMFs according to the Boltzmann relation. 
A

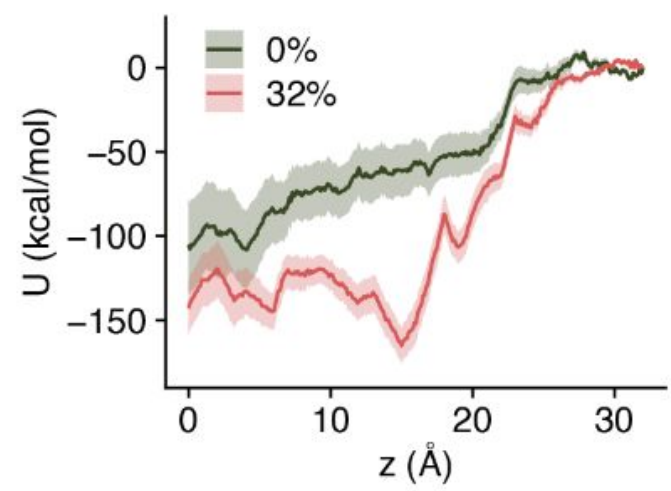

B

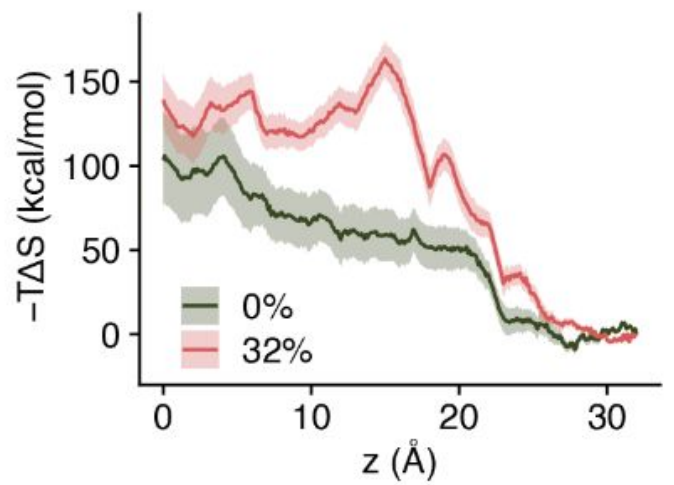

Figure S7. The enthalpy-entropy decomposition of the PMFs. Shown are the contributions from the internal energy (A) and the entropy (B) of two systems ( $x_{\text {chol }}=$ $0 \%$ in dark green line, and $x_{\text {chol }}=32 \%$ in red line) at $308 \mathrm{~K}$. 
A

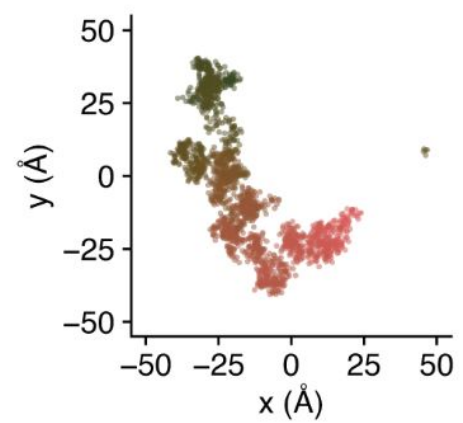

B

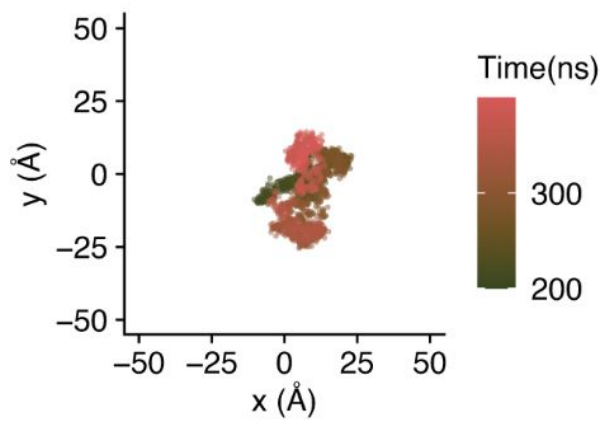

Figure S8. The $x-y$ positions of AMT in the lipid bilayer from the 200-400 ns portions of conventional MD simulations at $x_{c h o l}=0 \%$ (A) and $x_{c h o l}=32 \%$ (B). 